\title{
Acknowledgements to Referees
}

The editors wish to express their sincere appreciation to the numerous colleagues who have critically assessed submitted papers thereby providing the information indispensable for maintaining the scientific standing of Caries Research. J. M. ten Cate

P. A. Adriaens P. Anderson

B. Angmar-Månsson

T. Aoba

J. Arends R. R. Arnold

O. Backer Dirks R.A. Bagramian D.W. Banting

F. Barbakow

J.D.Beck

J. Beeley

D. Beighton

A. Bennick

R.J. Billings

D. Birkhed

A.S.Blinkhorn

J. M. P. M. Borggreven

J.J. ten Bosch

G.H.W. Bowden

W.H. Bowen
A. Boyde
G. Bradnock
D. Bratthall

A.L.J.J. Bronckers

J. Brunelle

C. Bruun

R. C. Burgess H. J. Busscher W.T.Butler

J.P.Carlos J. Carlsson V. Caslavska S. J. Challacombe C. L. Chow J. Christoffersen B.H.

Clarkson R.E. Corpron S.L. Creanor M.E.J. Curzon

C. Dawes

G.H. Dibdin

A. G. Dijkman

J. Disney

W. Distler H. Donoghue C.W.I. Douglas M.C. Downer

D. Drucker

R. M. Duckworth

J.E. Eastoe W.M.Edgar S. Edwardsson D.R. Eisenmann J. Ekstrand R.J. Elderton J.C.Elliott C.-

G. Emilson T Ericson 
J.D.B. Featherstone F. R. von der Fehr O. Fejerskov A.R. Firestone R.J. Fitzgerald L. Forsten R. Frank

D.A.M. Geddes

P.O.Glantz

J. de Graaff

T.H. Grenby

P. Grön

H. Grondahl

B. Guggenheim

I.R.Hamilton

R. Havenaar

D.I. Hay

M.L. Hayes

S. Higham

F. Hill

J.D.Hillman

P.N. Hirschman

M. Hobdell

J.S. van der Hoeven

W.P. Holbrook

P.J. Holloway

R. Holt

P. M.M. Hoppenbrouwers

K. Homer

H.S.Horowitz

T Imfeld G.S. Ingram

G.N. Jenkins C.L. Jones W. L. Jongebloed M. Joost Larsen S. Joyston-Bechal

H. Kalsbeek S. Kashket R. Katz E.Kay E.A.M. Kidd A.L. Kingman G. Koch K.G. König N.

Kotsanos T Koulourides

B. Krasse

K. P. Lang

S.A. Leach J.L. Leake R.Z. LeGeros T. Lehner M. A. Lennon D.H. Leverett R. Levine A. Linde L. Linder

C. van Loveren

H. Luoma

T. W. MacFarlane K.K. Mäkinen I.D.Mandel F. Manjii H. C. Margolis T.M. Marthaler H.C. van derMei J.R. Mellberg S.M.Mickalek L. Mikkelson

A. Milosevic

I.A.Mjör

T Modéer E.C. Moreno J.J. Murray

H. Nakagaki J.M. Navia D.G. A. Nelson E. Newbrun

B. Nyvad

B. Øgaard A. Oliveby D.M. O’Mullane

D.J.Page E.I.F. Pearce

N.B.Pitts J.J. Pollock S. Poulsen

V. Qvist 
H.R. Rawls

D.H. Retief

E.C. Reynolds

A. Richards

G. Roberts

P. Rock

A. H. Rogers

G. Rølla

A. J. Rugg-Gunn

R. R.B.Russell

H.J. Sandham C. A. Saxton M.J.M. Schaeken

F. Schäfer

A. A. Scheie

R. Schmid

P. Schüpbach

L.Shaw

A. Sheiham

R.P. Shellis

S.A. Shrestha-Mundorff

J. W. Simmelink

B.G.N. Smith

K.J.M. Söderholm

E. Soderling

J.J. de Soet

T. Sønju

C.J.Spak

K.W.Stephen

G.K. Stookey

R. Strang

G. Sundqvist

M. Svanberg

J.M. Tanzer A. Tatevossian J.O. Tenovuo E. Theilade A. Thylstrup R.S.Tobias L. Trahan S.

Tweetman

A. Veis

R.M.H. Verbeeck

E.H. Verdonschot

W. Wade

D. Watts

J.A. Weatherell

J.S.Wefel

S. H.Y.Wei

A. Wenzel

H. Whelton

R.A. Whiley

D.J. White

S. White 
G.M.Whitford

G.B. Winter

J.H.M. Wöltgens

H. V. Worthington

C.D.Wu-Yuan

D. Zero 